\title{
Selective maintenance optimisation for series- parallel systems alternating missions and scheduled breaks with stochastic durations
}

\author{
Abdelhakim Khatab, EL Houssaine Aghezzaf, Claver Diallo \& Imene Djelloul
}

To cite this article: Abdelhakim Khatab, EL Houssaine Aghezzaf, Claver Diallo \& Imene Djelloul (2017): Selective maintenance optimisation for series-parallel systems alternating missions and scheduled breaks with stochastic durations, International Journal of Production Research, DOI: 10.1080/00207543.2017.1290295

To link to this article: http://dx.doi.org/10.1080/00207543.2017.1290295

$$
\text { 里 Published online: } 21 \text { Feb } 2017 .
$$

Submit your article to this journal ¿

Q View related articles $\square$

View Crossmark data $\nearrow$ 


\title{
Selective maintenance optimisation for series-parallel systems alternating missions and scheduled breaks with stochastic durations
}

\author{
Abdelhakim Khatab $^{\mathrm{a} *}$, EL Houssaine Aghezzaf $^{\mathrm{b}}$, Claver Diallo $^{\mathrm{c}}$ (1) and Imene Djelloul ${ }^{\mathrm{d}}$ \\ ${ }^{a}$ Laboratory of Industrial Engineering, Production and Maintenance (LGIPM), National School of Engineering, Lorraine University, \\ Metz, France; ${ }^{b}$ Faculty of Engineering and Architecture, Department of Industrial Systems Engineering and Product Design, Ghent \\ University and Flanders Make, Ghent, Belgium, ${ }^{c}$ Industrial Engineering, Dalhousie University, Halifax, Nova Scotia, Canada; \\ ${ }^{d}$ Manufacturing Engineering Laboratory of Tlemcen (MELT), Abou Bekr Belkaid University of Tlemcen, Algiers, Algeria
}

(Received 4 December 2016; accepted 26 January 2017)

\begin{abstract}
This paper deals with the selective maintenance problem for a multi-component system performing consecutive missions separated by scheduled breaks. To increase the probability of successfully completing its next mission, the system components are maintained during the break. A list of potential imperfect maintenance actions on each component, ranging from minimal repair to replacement is available. The general hybrid hazard rate approach is used to model the reliability improvement of the system components. Durations of the maintenance actions, the mission and the breaks are stochastic with known probability distributions. The resulting optimisation problem is modelled as a non-linear stochastic programme. Its objective is to determine a cost-optimal subset of maintenance actions to be performed on the components given the limited stochastic duration of the break and the minimum system reliability level required to complete the next mission. The fundamental concepts and relevant parameters of this decision-making problem are developed and discussed. Numerical experiments are provided to demonstrate the added value of solving this selective maintenance problem as a stochastic optimisation programme.
\end{abstract}

Keywords: selective maintenance; reliability; preventive maintenance; stochastic optimisation

\section{Introduction}

In some businesses such as maritime and airline companies, systems operate according to an alternating sequence of missions and scheduled breaks. To prepare the system to successfully complete its next mission, its components must be properly maintained during the scheduled intermission break. Due to the limited duration of the scheduled breaks and scarce maintenance resources, only a limited set of components can be maintained during the breaks. It is therefore necessary to identify an optimal subset of components to maintain and the type of maintenance actions to be performed on these components to meet the predetermined reliability level required for the next mission. In the literature, this kind of maintenance strategy is known as the selective maintenance.

Selective maintenance was initially introduced by Rice, Cassady, and Nachlas (1998). The authors considered a seriesparallel system in which the subsystems are assumed to be composed of independent and identically distributed (i.i.d) components each having a lifetime that is also assumed to be exponentially distributed. The replacement of components at failure is the only maintenance option. To overcome the restrictive hypothesis of identical subsystem components in Rice, Cassady, and Nachlas (1998), the authors in Cassady, Pohl, and Murdock (2001) developed a more general framework for selective maintenance of a system whose reliability block diagram may be a combination of series, parallel and bridge structures.

Cassady, Murdock, and Pohl (2001) studied the selective maintenance problem in a series-parallel system, where the components have Weibull distributed lifetimes. For each component of the system three maintenance actions are considered: minimal repair, corrective replacement of a failed component and preventive replacement of a working component. The resulting selective maintenance problem is solved using an enumeration method. However, enumeration solution methods become rapidly unusable when the number of the system components increases. To deal with the combinatorial complexity arising with large-size systems, four improved enumeration procedures are proposed in Rajagopalan and Cassady (2006) to reduce the computation times. An exact method based on the branch-and-bound procedure and a Tabu search-based algorithm are proposed in Lust, Roux, and Riane (2009). Khatab, Ait-Kadi, and Nourelfath (2007) proposed two heuristic methods, adapted from those used to solve the redundancy allocation problem (Aggrawal 1976; Gopal, Aggrawal, and Gupta 1978;

*Corresponding author. Email: abdelhakim.khatab@univ-lorraine.fr 
Sharma and Venkateswaran 1971). Maaroufi, Chelbi, and Rezg (2013) studied the selective maintenance problem for a system where some components are subject to both global failure propagation and isolation.

Imperfect maintenance in the selective maintenance setting is addressed by Liu and Huang (2010), where the age reduction coefficient approach (Malik 1979) is used to model imperfect maintenance. The same imperfect selective maintenance model was studied in Zhu et al. (2011) and applied to a machining line system in the automotive industry. Panday et al. (2013) also studied the selective maintenance problem for binary systems under imperfect maintenance using the hybrid hazard rate approach introduced in Lin, Zuo, and Yam (2000). In Liu and Huang (2010) and Panday et al. (2013), a set of maintenance levels, ranging from minimal repair to replacement, are used to improve the reliability of a system component. Note that in Liu and Huang (2010) and Zhu et al. (2011), the only parameter that determines the improvement in the component's health is the age reduction coefficient. In Panday et al. (2013), however, both the age reduction coefficient and the adjustment coefficient impact the component's health. A more recent work, Khatab and Aghezzaf (2016) studied the selective maintenance problem when the quality of imperfect maintenance is stochastic. A non-linear and stochastic optimisation problem was proposed and solved for a series-parallel system.

Selective maintenance problem have also been investigated for multi-state systems (MSS) in Chen, Mend, and Zuo (1999) where system components and the system itself may be in more than two possible states. Liu and Huang (2010) considered MSS where components are characterised by two operating states and subject to imperfect maintenance that can bring the condition of a component to an intermediate level. Pandey, Zuo, and Moghaddass (2013) investigated the selective maintenance problem for a MSS where the functioning of each component is modelled as a continuous-time Markov chain with more than two states. Dao, Zuo, and Pandey (2014) and Dao and Zuo (2016) studied the selective maintenance problem while considering economic and stochastic dependencies among MSS components. Such dependency is also considered when dealing with the maintenance optimisation of MSS. In Zhou et al. (2015), several levels of imperfect maintenance are considered and the system is composed of multi-state components modelled as in Pandey, Zuo, and Moghaddass (2013). In a more recent paper, Dao and Zuo (2017) investigated the selective maintenance problem in a MSS setting where structural relationships between components are accounted for.

Won and Chung (2000) studied a maintenance policy for a system under a random time horizon. The system is minimally repaired at failure and replaced by a new one whenever its age reaches a specified value. Conditions under which the optimal age replacement exists are derived for the particular case where the system lifetime is Weibull distributed. This results is generalised in Khatab, Rezg, and Ait-Kadi (2011a, 2011b), where the authors proposed a maintenance optimisation model for a system operating under a random time horizon with general lifetime distributions. These papers view the system as a single units operating continuously over time and the only available imperfect maintenance action is the minimal repair. Times required toperform either replacement or a minimal repair actions are not accounted for. Consequently, maintenance resources constraints are not addressed in these papers.

The selective maintenance problems addressed up until now assume that the durations of the breaks and missions are deterministic and are known in advance. This assumption may however not be valid in many real-world situations, where it is difficult to estimate the exact duration of the following missions. The duration of a mission may indeed be impacted by the occurrence of unexpected events causing the system to either abort the mission or continue operating at lower (higher) speed/rate hence increasing (decreasing) the mission duration. For example, weather conditions can randomly change favourably or unfavourably causing a ship to take less or more time than expected before returning to the dock. In addition to the density of water on which the displacement is performed, vessel and ship motion also varies depending on the type and quantity of the freight being transported. This may increase or shorten the duration of the mission. Also, unexpected events such as air-traffic jams, weather, strikes at airports may cause the rerouting of aircraft thus resulting in possible increase or decrease the flight duration. The duration of a break is also difficult to estimate. Adverse sudden changes in the weather conditions may lead to the delaying of an aircraft take-off or the departure of ships from the port. In freight transport, the departure of a ship may be impacted by the traffic density and infrastructure (river locks and hydroelectric dams) management. It is probably more realistic to consider that missions as well as breaks durations are not usually known in advance, but are rather random variables governed by some appropriate probability distributions. The only existing works that the authors are aware of appeared in Djelloul et al. (2015) and Khatab et al. (2016). In Djelloul et al. (2015), the mission duration of the system is uncertain and modelled as a random variable while the break is considered deterministic with a constant duration. The selective maintenance model in Khatab et al. (2016) extends the model proposed in Djelloul et al. (2015) and considers the durations of both the mission and the break to be stochastic. The resulting selective maintenance optimisation formulations are therefore non-linear and stochastic.

This paper extends the shorter preliminary work presented at a conference Khatab et al. (2016). In addition to the stochastic durations of missions and break, the present paper considers that the times spent on maintenance actions are also stochastic. Furthermore, the more general hybrid hazard rate model is used to characterise the reliability improvement for the system components. We have also established a relationship between the cost induced by a maintenance action and the level of that 
maintenance action. The same relationship is developed to relate the time spent on a maintenance action and the level of that maintenance action. These relationships are formally defined as functions of the mean residual useful lifetimes of the system components. More details are also provided and additional numerical experiments are conducted to demonstrate the added value and the validity of the proposed approach. The selective maintenance problem to be solved consists first in selecting a subset of components to be maintained and then choosing the level of maintenance to be performed on each component. The problem is formulated as a non-linear stochastic optimisation problem where probability distributions assigned to durations of the maintenance actions, the missions and the breaks are fully accounted for. The objective function is either to maximise the reliability for the next mission while taking the maintenance budget and duration of the break into account, or to minimise the total maintenance cost subject to required reliability level and maintenance duration during the break, or to minimise the total maintenance duration subject to the required reliability level and maintenance budget. This paper focuses on the second objective function and presents an elegant and comprehensive formulation for this selective maintenance optimisation problem.

The following assumptions are considered in this paper:

(1) The system consists of multiple, repairable binary components (the components and the system are either functioning or failed).

(2) During the break, system components do not age, i.e. the age of a component is operation-dependent.

(3) No maintenance activity is allowed during the mission. Maintenance activities are allowed only during the break.

(4) After a replacement, a component becomes as good as new.

(5) After a minimal repair, a component becomes as bad as old.

(6) A list of imperfect maintenance levels are also available. An imperfect maintenance action when performed on a component brings its health condition back between as good as new and as bad as old.

(7) All required limited resources (budget, repairman and time) are available when needed for maintenance activities, and they are known and fixed. Here, only one repairman is available.

The remainder of the paper is organised as follows. Section 2 describes the system under consideration and defines its mission profile. Section 3 gives a brief background on the hybrid hazard rate approach for modelling imperfect maintenance. Section 3 describes the maintenance levels and defines the maintenance duration and cost functions. The expression of the probability of mission completion is developed in Section 4. Sections 5 present a formulation of the stochastic selective maintenance optimisation problem (SSMOP) and discuss some of its major properties. Numerical experiments are provided in Section 6 to show how the stochasticity of the durations of the mission, the break and the maintenance actions impact the maintenance decisions. Conclusions are drawn and future extensions are discussed in Section 7.

\section{Description of the system under consideration}

Without loss of generality, the selective maintenance problem addressed in the present work concerns a series-parallel system $S$ composed of $n$ series subsystems $S_{i}(i=1, \ldots, n)$ each of which is composed of $N_{i} s$-independent, and possibly, nonidentical components/parts $P_{i j}\left(j=1, \ldots, N_{i}\right)$ arranged in parallel. The system is assumed to have just finished a mission and then, turned off during the scheduled break of finite length and becomes available for possible maintenance activities. The system is thereafter used to execute the next mission of a given duration. The duration of the scheduled break is denoted as $D$ and the duration of the next mission is denoted by $U$. In the present work, these duration are considered stochastic rather than deterministic. Therefore, durations $D$ and $U$ are modelled as random variables governed by their respective probability density functions (pdf) and the cumulative distribution functions (cdf). The pdf and cdf corresponding to duration $D$ are, respectively, denoted as $f_{D}(t)$ and $F_{D}(t)$ and defined on the support [ $\left.D_{\min }, D_{\max }\right]$. The pdf and cdf assigned to duration $U$ are defined on the support $\left[U_{\min }, U_{\max }\right]$ and, respectively, denoted as $f_{U}(t)$ and $F_{U}(t)$.

At the end of the current mission, the state variable $X_{i j}$ describes the status of the component $P_{i j}$. Similarly, the state variable $Y_{i j}$ describes the status $P_{i j}$ at the beginning of the next mission. These two state variables are then formally defined as:

$$
\begin{aligned}
X_{i j} & =\left\{\begin{array}{l}
1, \text { if } P_{i j} \text { is functioning at the end of the current mission, } \\
0, \text { otherwise. }
\end{array}\right. \\
Y_{i j} & =\left\{\begin{array}{l}
1, \text { if } P_{i j} \text { is functioning at the beginning of the next mission, } \\
0, \text { otherwise. }
\end{array}\right.
\end{aligned}
$$

When running the current mission, the failure rate of the component $P_{i j}$ is denoted as $h_{i j}(t)$. At the end of the current mission, the age of each component $P_{i j}$ is denoted by $A_{i j}$. We also denote by $B_{i j}$, the age of $P_{i j}$ at the beginning of the next mission. If maintenance is not performed on $P_{i j}$, then $A_{i j}=B_{i j}$, according to assumption (2). 


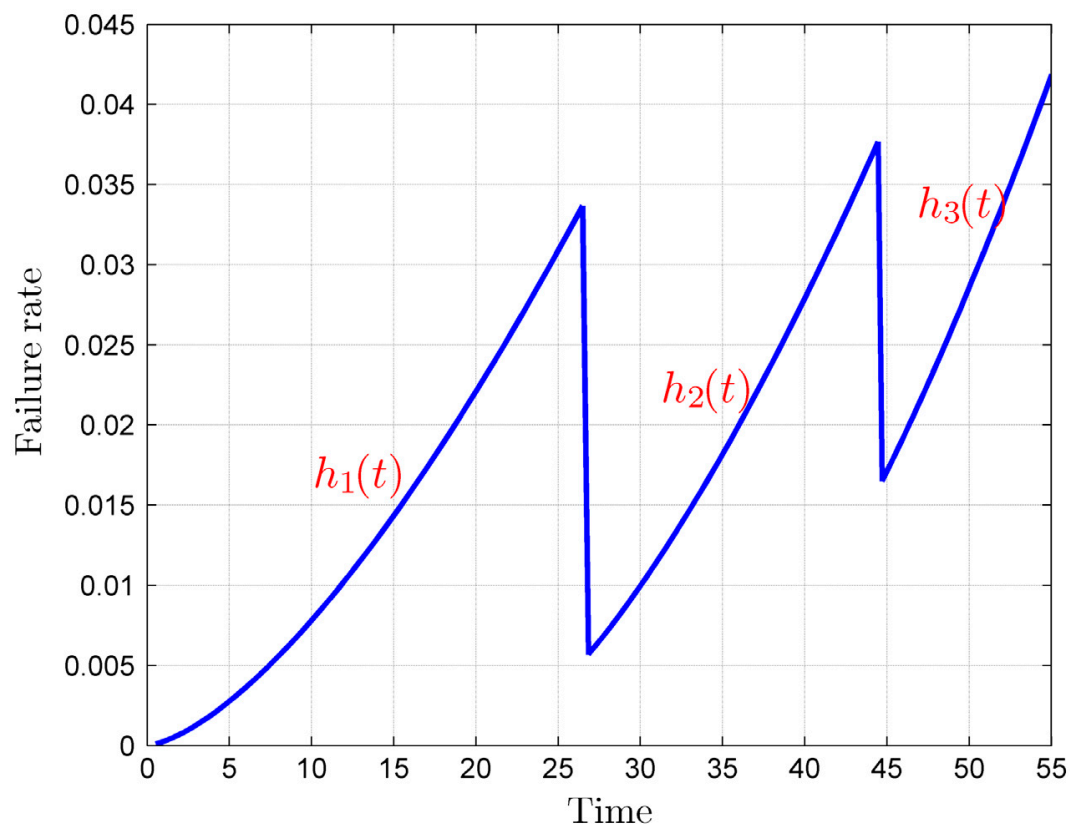

Figure 1. Profile of the system's failure rate evolving under the hybrid hazard rate model

\section{Imperfect maintenance model, cost and duration functions}

In production and service systems, it is often required to minimise interruptions due to machine failures. Maintenance actions are performed on systems in order to avoid failures and costly downtime. As noted in Lin, Zuo, and Yam (2000), traditional maintenance models assume that the system after a maintenance is either as good as new (i.e. perfect maintenance or replacement) or as bad as old (i.e. minimal repair). The more realistic and generalised approach is to assume that the system after maintenance lies somewhere between as good as new and as bad as old, which is called imperfect maintenance. For example, opening a machine to repair one component may result in damaging other components leading to an increase of the slope of the failure rate function. Another reason is that lack of resources or time may not permit to maintain the system as best as possible. As a consequence, maintenance actions occur at an increased frequency (i.e. the intervals between consecutive maintenance actions reduce as time passes as shown in Figure 1). At one point, these maintenance actions are so frequent that it becomes better to replace the system rather than continuing to carry out maintenance. The authors in Pham and Wang (1996) and Wang (2002) provided a review of early existing imperfect maintenance models. Among these models are the age reduction model of Malik Malik (1979), the adjustment coefficient model of Nakagawa Nakagawa (1988), Nakagawa and Mizutani (2009) where the hazard rate is increased after each maintenance action, the hybrid hazard rate model of Lin, Zuo, and Yam (2000) which is later used in Lin, Zuo, and Yam (2001), El-Ferik and Ben-Daya (2006), Khatab (2015), Sheu and Chang (2009), the quasi-renewal process model (Park and Pham 2010; Wang and Pham 1999, the geometric process model (Lam 1988), and the Kijima's model based on the generalised renewal process (Kijima, Murimura, and Suzuki 1988; Kijima 1989). More details on models and methodologies available to deal with imperfect maintenance can be found in Nakagawa (2008) and Wang and Pham (2006). An extensive review of Kijima's model and its applications can be found in Tanwar, Rai, and Bolia (2014).

In the present work, the imperfect maintenance model used is based on the hybrid hazard rate approach initially proposed by Lin, Zuo, and Yam (2000) and later used by Khatab (2015), El-Ferik and Ben-Daya (2006), Sheu and Chang (2009) and many others. The hybrid hazard rate approach proposed by Lin, Zuo, and Yam (2000) combines failure rate adjustment and age reduction approaches. It is a more general and realistic case as it allows the maintenance action to reduce the effective age to a certain value but also to change the slope of the hazard rate function. Indeed, if the hazard rate function of the system was $h(t)$ just before performing a maintenance at time $t$, then it becomes $\xi h(\theta t+x)$ right after the maintenance, where $\xi \geq 1$, is the hazard rate adjustment coefficient, $0 \leq \theta \leq 1$ is the age reduction coefficient and $x \geq 0$ represents the time elapsed from the instant of the maintenance completion. When $\xi=1$, the hybrid model reduces to age reduction model, while it reduces to hazard rate adjustment model when $\theta=0$. The age reduction and the adjustment coefficients are usually 
estimated from subject matter experts suggestion or from reliability and maintenance historical data (Malik 1979; Canfield 1986; Wu and Clements-Croome 2005).

\subsection{Maintenance levels and their costs}

To perform maintenance activities, a list of $L_{i j}+1$ potential maintenance levels/options $1, l \in\left\{0,1, \ldots, L_{i j}\right\}$, are available for each component $P_{i j}$. Level $l=0$ corresponds to the "Do nothing" case, i.e. no maintenance is performed on $P_{i j}$. Level $l=1$ corresponds to the minimal repair maintenance action which, when performed, brings the component to an as bad as old condition. Level $l=L_{i j}$ corresponds to the replacement case, after which the component becomes as good as new. Other intermediate values of $1<l<L_{i j}$ represent imperfect maintenance actions such that when performed they bring the component's condition between the as good as new and as bad as old conditions. Each maintenance level $l \geq 1$ available for component $P_{i j}$ is assigned two coefficients: the age reduction coefficient $\theta_{i j l}$ and a failure rate adjustment coefficient $\xi_{i j l}$. According to the hybrid hazard rate model, when a maintenance action of level $l \geq 1$ is performed on $P_{i j}$, at the end of the current mission, it reduces its age from $A_{i j}$, to $\theta_{i j l} A_{i j}$ and its failure rate becomes $\xi_{i j l} h_{i j}\left(t+\theta_{i j l} A_{i j}\right)$, for $t \geq 0$.

For the two particular maintenance levels $l=1$ and $l=L_{i j}$, the age reduction and the adjustment coefficients are such that $\theta_{i j 1}=1, \theta_{i j, L_{i j}}=0$, and $\xi_{i j 1}=\xi_{i j, L_{i j}}=1$. In the present work, both corrective maintenance (CM) and preventive maintenance (PM) are modelled according to the hybrid hazard rate model, i.e. both maintenance types can be imperfect.

\subsection{Maintenance cost model}

The maintenance cost $C_{i j l}$ induced by a maintenance action of level $l \in\left\{1, \ldots, L_{i j}\right\}$ when performed on component $P_{i j}$ is a PM cost $C_{i j l}^{p}$ if $P_{i j}$ is still functioning at the end of the current mission, or is a CM cost $C_{i j l}^{c}$ if $P_{i j}$ has failed at the end of the current mission with a common assumption that $C_{i j l}^{c}>C_{i j l}^{p}$. This maintenance cost is then defined as:

$$
C_{i j l}= \begin{cases}C_{i j l}^{p}, & \text { if } X_{i j}=1 \text { and } l>1 \\ C_{i j l}^{c}, & \text { if } X_{i j}=0\end{cases}
$$

For $l=1$ (minimal repair), the minimal repair cost is denoted by $C_{i j}^{m r}$. Similarly, for a failed component, the replacement cost (level $l=L_{i j}$ ) is denoted by $C_{i j, L_{i j}}^{c}$. In case the component is still functioning at the end of the current mission, the cost to preventively replace it with a new one (level $l=L_{i j}$ ) is denoted by $C_{i j, L_{i j}}^{p}$. It should be noted that minimal repair (level $l=1$ ) is not available for PM actions. induced by preventive replacement of component $P_{i j}$ is defined as $M C_{i j}^{p, L_{i j}}=R C_{i j}^{p}$, while $M C_{i j}^{p, 1}$ is not eligible (i.e. minimal repair is admissible as a maintenance option only for a failed component). Maintenance costs $C_{i j}^{m r}, C_{i j, L_{i j}}^{c}$, and $C_{i j, L_{i j}}^{p}$ are assumed to be constant and known. However, the maintenance costs of intermediate maintenance level $1<l<L_{i j}$ are defined in terms of the maintenance costs these extreme cases. Hence, we have:

$$
\begin{aligned}
& C_{i j l}^{p}=C_{i j}^{p} \cdot\left(1-\theta_{i j l}\right)^{\delta_{i j}\left(A_{i j}\right)}, \\
& C_{i j l}^{c}=C_{i j}^{c} \cdot\left(1-\theta_{i j l}\right)^{\delta_{i j}\left(A_{i j}\right)} .
\end{aligned}
$$

In the above cost structures, the parameter $\delta_{i j}\left(A_{i j}\right)$ is introduced as a characteristic function to establish a relationship between the maintenance cost and the age $A_{i j}$ of the component $P_{i j}$ at the end of a mission. In line with the idea discussed in Khatab and Aghezzaf (2016), the formulation of $\delta_{i j}\left(A_{i j}\right)$ is proposed to reflect the relationship between the reliability of a component and its effective age. Equations (4) and (5) enable the modelling of the fact that for the same maintenance level, the maintenance cost of a younger system is less than that of an older system. Thus, $\delta_{i j}\left(A_{i j}\right)$ is a function of the age $A_{i j}$ and defined as the ratio of the mean residual lifetime $\operatorname{MRUL}\left(A_{i j}\right)$ of component $P_{i j}$ and its age $A_{i j}$ :

$$
\delta_{i j}\left(A_{i j}\right)=\frac{\operatorname{MRUL}\left(A_{i j}\right)}{A_{i j}} .
$$

The mean residual useful lifetime $\operatorname{MRUL}\left(A_{i j}\right)$ of the component $P_{i j}$ whose failure rate $h_{i j}(t)$ is then given by:

$$
\operatorname{MRUL}\left(A_{i j}\right)=\int_{A_{i j}}^{\infty} \exp \left(-\int_{A_{i j}}^{t} h_{i j}(x) \mathrm{d} x\right) \mathrm{d} t
$$




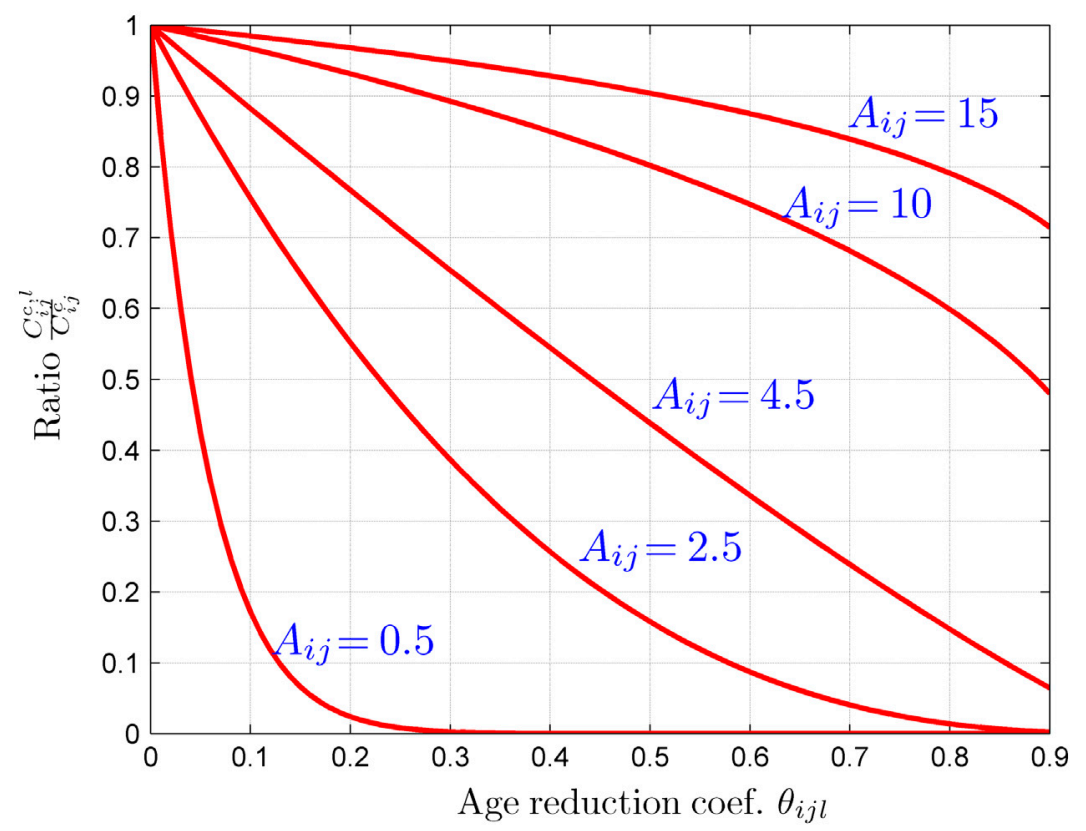

Figure 2. The ratio $\frac{C_{i j l}^{c}}{C_{i j}^{c}}$ vs. the age reduction coefficient $\theta_{i j l}$

The characteristic function $\delta_{i j}\left(A_{i j}\right)$ is then defined as:

$$
\delta_{i j}\left(A_{i j}\right)=\frac{\int_{A_{i j}}^{\infty} \exp \left(-\int_{A_{i j}}^{t} h_{i j}(x) \mathrm{d} x\right) \mathrm{d} t}{A_{i j}} .
$$

If the component's age $A_{i j}$ is less than its $\operatorname{MRUL}\left(A_{i j}\right)$, it is said to be relatively younger, then its corresponding $\delta_{i j}\left(A_{i j}\right)$ is high and because $0 \leq \theta_{i j l}<1$, its induced maintenance cost from Equations (4) and (5) is smaller. Similarly, if component's age $A_{i j}$ is higher than its $\operatorname{MRUL}\left(A_{i j}\right)$, its corresponding maintenance cost will be significantly higher. As stated above, Equations (4) and (5) enable the modelling of the fact that for the same maintenance level, the maintenance cost of a younger system is less than that of an older system.

To show how the characteristic function $\delta_{i j}\left(A_{i j}\right)$ impacts the maintenance cost of a component $P_{i j}$, let us assume that the lifetimes of the components follow a Weibull distribution with shape and scale parameters set, respectively, to 2.3 and 10. For different values of the initial age $A_{i j}$, Figure 2 gives the ratio $\frac{C_{i j l}^{c}}{C_{i j}^{c}}$ vs. the age reduction $\theta_{i j l}$. From this figure, one may indeed observe that for a fixed value of the age reduction coefficient, the maintenance cost increases as the initial age $A_{i j}$ increases. This observation supports the assumption according to which the cost resulting from maintenance performed on a younger component is cheaper than the same maintenance level performed on an older component.

\subsection{Expected maintenance durations}

By analogy to the component maintenance cost, the maintenance duration $T_{i j l}$ of a component $P_{i j}$ is defined by:

$$
T_{i j l}= \begin{cases}T_{i j l}^{p}, & \text { if } X_{i j}=1 \text { and } l>1, \\ T_{i j l}^{c}, & \text { if } X_{i j}=0 .\end{cases}
$$

Similar to the maintenance cost, particular values of maintenance durations are also defined for $l=1$ (minimal repair) and $l=L_{i j}$ (replacement). For a failed component, the duration of a minimal repair is $T_{i j}^{m r}$ when the duration of a replacement is $T_{i j, L_{i j}}^{c}$. In the case where the component is working at the end of the mission, the duration of the replacement is $T_{i j, L_{i j}}^{p}$ while there is no minimal repair.

As pointed out in our previous discussion, time spent to perform maintenance activities is always subject to variability due, for example, to the operating environment, maintenance operator experience, etc. For accurate modelling and reliability 
assessment, this variability should be accounted for in the maintenance modelling process in general, and in selective maintenance in particular. In the present work, times spent performing maintenance actions are considered as random variables governed by appropriate probability distributions. Therefore, durations $T_{i j l}^{c}$ and $T_{i j l}^{p}$ are random variables and their cdfs are denoted by $G_{i j l}^{c}(t)$ and $G_{i j l}^{p}(t)$, respectively.

The expected duration of maintenance of level $l\left(1<l<L_{i j}\right)$ on component $P_{i j}$ is also function of both the age reduction coefficient assigned to $l$ and the MRUL of the component. Formally, the expected durations of an intermediate maintenance action of level $1<l<L_{i j}$ are therefore defined as:

$$
\begin{aligned}
& \mathbb{E}\left[T_{i j l}^{p}\right]=\mathbb{E}\left[T_{i j}^{p}\right]\left(1-\theta_{i j l}\right)^{\delta_{i j}\left(A_{i j}\right)}, \\
& \mathbb{E}\left[T_{i j l}^{c}\right]=\mathbb{E}\left[T_{i j}^{c}\right]\left(1-\theta_{i j l}\right)^{\delta_{i j}\left(A_{i j}\right)},
\end{aligned}
$$

where $\delta_{i j}\left(A_{i j}\right)$ is defined as in Equation (8).

\section{Reliability during the next mission}

According to the hybrid hazard rate imperfect maintenance model, if a maintenance action with an eligible level $l \in$ $\left\{1, \ldots, L_{i j}\right\}$ is performed on component $P_{i j}$ at the end of the current mission, the effective age $B_{i j}$ of $P_{i j}$ at the beginning of the next mission is $B_{i j}=\theta_{i j l} A_{i j}$, and its corresponding failure rate becomes:

$$
h_{i j l}(t)=\xi_{i j l} h_{i j}\left(\theta_{i j l} A_{i j}+t\right), \quad \text { for } t \geq 0 .
$$

At the end of the current mission, the operating state $X_{i j}$ as well as the age $A_{i j}$ are known for every component $P_{i j}$. Depending on the maintenance action performed on component $P_{i j}$, its corresponding operating state variable $Y_{i j}$ at the beginning of the next mission is updated according to Equation (2). Let $\mathcal{R}_{i j}^{c}$ be the conditional reliability that component $P_{i j}$ successfully completes, the next mission given that it received a maintenance action of level $l$ at the end of the current mission. This reliability depends on both effective age $B_{i j}$ of $P_{i j}$ at the beginning of the next mission as well as on the duration $U$ of this mission. From Equation (12) and taking into account the fact that duration $U$ is stochastic and governed by the cdf $F_{U}(t)$ on the support $\left[U_{\min }, U_{\max }\right]$, the conditional reliability $\mathcal{R}_{i j}^{c}$ is given by:

$$
\begin{aligned}
\mathcal{R}_{i j}^{c} & =\int_{U_{\min }}^{U_{\max }} \exp \left[-\int_{0}^{u} h_{i j l}(t) \mathrm{d} t\right] \mathrm{d} F_{U}(u) \\
& =\int_{U_{\min }}^{U_{\max }} \exp \left[-\xi_{i j l} \int_{\theta_{i j l} A_{i j}}^{\theta_{i j l} A_{i j}+u} h_{i j}(t) \mathrm{d} t\right] \mathrm{d} F_{U}(u) .
\end{aligned}
$$

The reliability $\mathcal{R}^{c}$ of the whole system during the next mission is evaluated from components reliabilities $\mathcal{R}_{i j}^{c}$ according to the system's reliability block diagram. In our case, components are arranged in a series-parallel configuration. Hence, the conditional reliability of subsystem $S_{i}(i=1, \ldots, n)$ and that of the system $S$, respectively, denoted by $\mathcal{R}_{i}^{c}$ and $\mathcal{R}^{c}$ for the next mission are given by:

$$
\begin{aligned}
& \mathcal{R}_{i}^{c}=1-\prod_{j=1}^{N_{i}}\left[1-\mathcal{R}_{i j}^{c} \cdot Y_{i j}\right], \text { and } \\
& \mathcal{R}^{c}=\prod_{i=1}^{n} \mathcal{R}_{i}^{c} .
\end{aligned}
$$

To illustrate how the stochasticity of the mission duration impacts the system reliability, we consider the following single component $P_{i j}$, for which the corresponding age $A_{i j}$, at the end of the current mission, is measured and assumed to be $A_{i j}=10$ units of time. The failure rate $h_{i j}(t)=\left(\frac{\beta_{i j}}{\eta_{i j}}\right)\left(\frac{t}{\eta_{i j}}\right)^{\beta_{i j}-1}$ of component $P_{i j}$ is assumed to be derived from a Weibull distributed lifetime with shape and scale parameters, respectively, equal to $\beta_{i j}=1.5$ and $\eta_{i j}=15$. Let us consider the case where the duration $U$ of the next mission is governed by a normal distribution defined on the truncated support [7,12]. That is, the system will operate the next mission for an interval of time which is normally distributed and lies between $U_{\min }=7$ and $U_{\max }=12$ units of time. The reliability to successfully perform the next mission is evaluated in two cases. In the first case, the distribution governing the duration $U$ is assumed to be truncated from $\mathcal{N}(8,1.5)$ whose pdf curve is drawn in Figure 3. The second case is the deterministic case where the next mission duration is assumed to be of constant duration and equal to 8 units of time. 


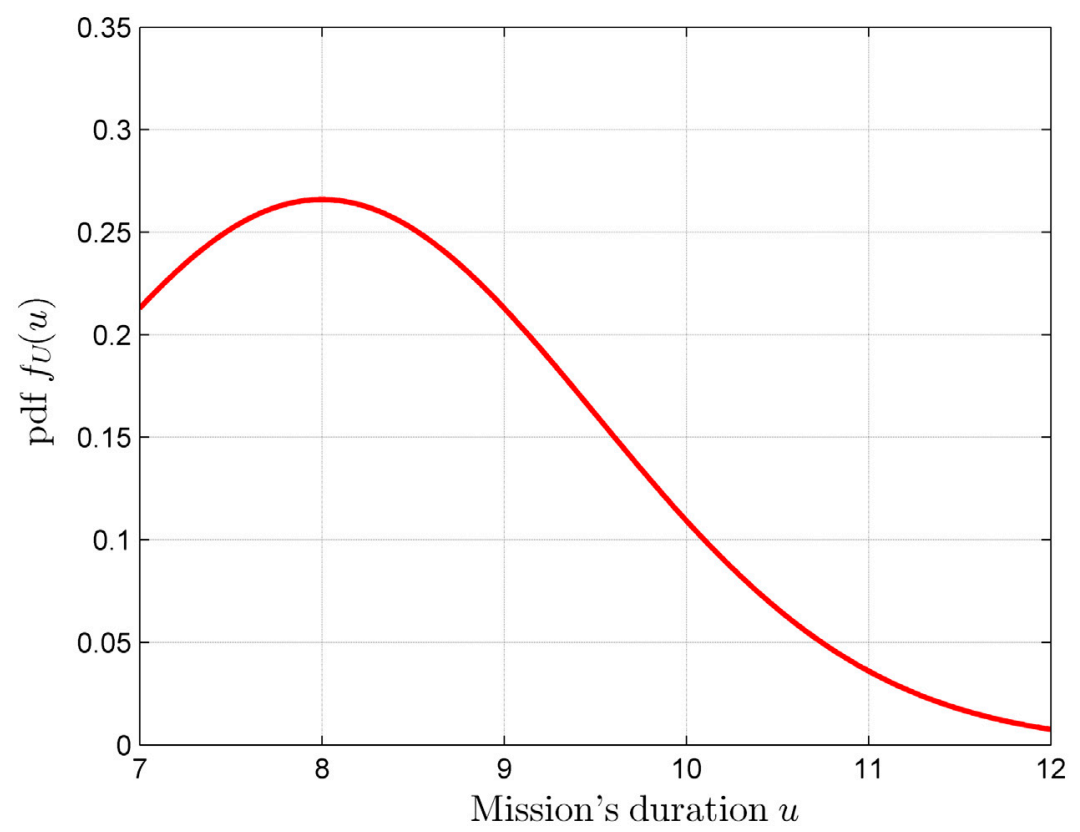

Figure 3. Truncated normal distribution: pdf of the next mission duration.

Let us assume that component $P_{i j}$ is still functioning at the end of the current mission (i.e. $X_{i j}=1$ ). If it is assumed, without loss of generality, that no maintenance is performed on $P_{i j}$ during the break, then in the deterministic case (i.e. $U=8$ ) $\mathcal{R}_{i j}^{c}=46.29 \%$. However, in the stochastic case, $(U$ is normal truncated from $\mathcal{N}(8,1.5)), \mathcal{R}_{i j}^{c}=43.45 \%$, which is $2.84 \%$ less than the reliability value obtained with the deterministic approach. We conclude that the deterministic approximation of the mission duration overestimates the real value of the system reliability. This result show that assuming mission durations to be deterministic rather than stochastic may lead to undesirable economic consequences and safety issues. The reliability computed using the deterministic duration may be either smaller or greater than the reliability obtained with the stochastic duration. In the first case, i.e. when the reliability based on the deterministic assumption is small, the actual reliability of the system obtained by considering stochastic duration is underestimated. This means that the maintenance plan suggested in the deterministic case, calls for unnecessary and more expensive levels of maintenance, thus unreasonably increasing the expected total cost of maintenance. However, if the reliability based on deterministic durations is higher, the actual reliability of the system is overestimated. This means that the maintenance plan suggested in the deterministic case may result in a high risk of not completing the next mission with the required minimum reliability level.

\section{The SSMOP}

Considering that the system has just survived the current mission, components may undergo maintenance activities. However, not all components may possibly be maintained due to the limitation on both maintenance budget and duration. Consequently, a selective maintenance problem must be solved. The objective is then to minimise the expected total maintenance cost taking into account, on one hand, the required minimum system reliability $\mathcal{R}_{0}$ to successfully completing the next mission, and the limited duration of the break, on the other hand. The probability of completing the next mission is obtained from the conditional reliability $\mathcal{R}^{c}$ computed in the previous section. To evaluate the total cost induced by maintenance actions and the corresponding total maintenance duration, the following decision variable $z_{i j l}$ is defined.

$$
z_{i j l}=\left\{\begin{array}{l}
1, \text { if maintenance level } l \text { is performed on } P_{i j} \\
0, \text { otherwise. }
\end{array}\right.
$$

The total cost of maintenance during the break is denoted by $T M C$ and computed as:

$$
T M C=P M C+C M C,
$$


where $P M C$ and $C M C$ denote the total cost induced by, respectively, PM and $\mathrm{CM}$ actions performed during the break. The total cost of PM actions is given by:

$$
P M C=\sum_{i=1}^{n} \sum_{j=1}^{N_{i}} \sum_{l=2}^{L_{i j}} C_{i j l}^{p} \cdot X_{i j} \cdot z_{i j l},
$$

where a PM action of level $l>1$ is allowed to be performed on component $P_{i j}$ only if $P_{i j}$ is still functioning at the end of the current mission, i.e. $X_{i j}=1$. By analogy, the total cost induced by CM actions is obtained from:

$$
C M C=\sum_{i=1}^{n} \sum_{j=1}^{N_{i}} \sum_{l=1}^{L_{i j}} C_{i j l}^{c} \cdot\left(1-X_{i j}\right) \cdot z_{i j l},
$$

where $\left(1-X_{i j}\right)$ states that the $\mathrm{CM}$ actions are available only for failed components.

The total time required to perform maintenance actions during the break is also composed of PM an CM durations denoted, respectively, by $P M T$ and $C M T$. These quantities are:

$$
\begin{aligned}
P M T & =\sum_{i=1}^{n} \sum_{j=1}^{N_{i}} \sum_{l=2}^{L_{i j}} T_{i j l}^{p} \cdot X_{i j} \cdot z_{i j l}, \\
C M T & =\sum_{i=1}^{n} \sum_{j=1}^{N_{i}} \sum_{l=1}^{L_{i j}} T_{i j l}^{c} \cdot\left(1-X_{i j}\right) \cdot z_{i j l} .
\end{aligned}
$$

Hence, the total time spent in maintenance during the break denoted by TMT is the sum of the PM and CM durations: TMT $=P M T+C M T$.

The SSMOP is formulated as:

$$
\operatorname{Min}\left(\begin{array}{l}
\sum_{i=1}^{n} \sum_{j=1}^{N_{i}} \sum_{l=1}^{L_{i j}} C_{i j l}^{c}\left(1-X_{i j}\right) z_{i j l} \\
\left.+\sum_{i=1}^{n} \sum_{j=1}^{N_{i}} \sum_{l=2}^{L_{i j}} C_{i j l}^{p} X_{i j} z_{i j l}\right)
\end{array}\right)
$$

Subject to:

$$
\begin{aligned}
& \mathcal{R}^{c} \geq \mathcal{R}_{0}, \\
& \operatorname{Pr}(\mathrm{TMT} \leq D)>\tau_{s}, \\
& \sum_{l=1}^{L_{i j}}\left(1-X_{i j}\right) z_{i j l}+\sum_{l=2}^{L_{i j}} X_{i j} z_{i j l} \leq 1, \\
& z_{i j 1} \leq 1-X_{i j}, \\
& Y_{i j}=X_{i j}+\sum_{l=1}^{L_{i j}}\left(1-X_{i j}\right) z_{i j l}, \\
& B_{i j}=A_{i j}\left[\sum_{l=1}^{L_{i j}} \theta_{i j l} z_{i j l}+\left(1-\sum_{l=1}^{L_{i j}} z_{i j l}\right)\right], \\
& z_{i j l} \in\{0,1\} ; i \in\{1, \ldots, n\} ; j \in\left\{1, \ldots, N_{i}\right\} ; l \in\left\{1, \ldots, L_{i j}\right\} .
\end{aligned}
$$

In the above optimisation model, Equations (23) and (24) are, respectively, the required reliability level and the maintenance duration constraints. Constraint (24) is a chance constraint requiring careful treatment. Given that both the durations of maintenance actions and the break are stochastic, there will always be a risk that the expected total duration of all maintenance activities may exceed the duration of the break. Hence, a service level ratio $\tau_{s}$ is introduced to quantify the proportion of times (or probability) that the maintenance actions are completed within the break. The risk corresponding to the inability to perform a selected maintenance plan is then assessed at $1-\tau_{s}$. For each component $P_{i j}$, Equations (25) states that only one maintenance level can be selected if the component is to be maintained. Constraint (26) states that minimal repair is eligible only on a failed component. Constraint (27) updates the operating ages of components. If a level $l\left(1 \leq l \leq L_{i j}\right)$ maintenance is selected then the age of the component is reduced by the corresponding $\theta_{i j l}$. If no level $l$ maintenance is selected, then the age does not change. For a given system configuration, this stochastic optimisation problem can be solved using the usual 
stochastic optimisation techniques (see e.g. Birge and Louveaux 1997; Spall 2005). In the following section, we provide two experiments and fully discuss how the stochasticity of the mission and break durations as well as maintenance durations may impact the maintenance level selection decisions.

\section{Numerical experiments}

To perform the subsequent experiments, we consider that PM and CM durations $T_{i j l}^{p}$ and $T_{i j l}^{c}\left(l=1, \ldots, L_{i j}\right)$ are governed by $\operatorname{Gamma}$ distributions $\operatorname{Gam}\left(\alpha_{i j l}^{p}, \zeta_{i j l}^{p}\right)$ and $\operatorname{Gam}\left(\alpha_{i j l}^{c}, \zeta_{i j l}^{c}\right)$, respectively. Their respective pdfs are $g_{i j l}^{p}(t)=\frac{\mathrm{d} G_{i j l}^{p}(t)}{\mathrm{d}}$ and $g_{i j l}^{c}(t)=\frac{\mathrm{d} G_{i j l}^{c}(t)}{\mathrm{d}}$ such that:

$$
\begin{aligned}
g_{i j l}^{p}(t) & =\frac{t^{\alpha_{i j l}^{p}-1}}{\Gamma\left(\alpha_{i j l}^{p}\right)\left(\zeta_{i j l}^{p}\right)^{\alpha_{i j l}^{p}}} \exp \left(-\frac{t}{\zeta_{i j l}^{p}}\right), \\
g_{i j l}^{c}(t) & =\frac{t^{\alpha_{i j l}^{c}-1}}{\Gamma\left(\alpha_{i j l}^{c}\right)\left(\zeta_{i j l}^{c}\right)^{\alpha_{i j l}^{c}}} \exp \left(-\frac{t}{\zeta_{i j l}^{c}}\right),
\end{aligned}
$$

where $\alpha_{i j l}^{p}$ and $\alpha_{i j l}^{c}$ are the shape parameters, $\zeta_{i j l}^{p}$ and $\zeta_{i j l}^{c}$ are the scale parameters, and $\Gamma(s)=\int_{0}^{\infty} x^{s-1} \exp (-x) \mathrm{d} x$ is the Gamma function. The cdfs $G_{i j l}^{p}(t)$ and $G_{i j l}^{c}(t)$ are then obtained as:

$$
\begin{aligned}
& G_{i j l}^{p}(t)= \frac{\Gamma\left(\alpha_{i j l}^{p}, \frac{t}{\zeta_{i j l}^{p}}\right)}{\Gamma\left(\alpha_{i j l}^{p}\right)}, \\
& G_{i j l}^{c}(t)=\frac{\Gamma\left(\alpha_{i j l}^{c}, \frac{t}{\zeta_{i j l}^{c}}\right)}{\Gamma\left(\alpha_{i j}^{c, l}\right)} .
\end{aligned}
$$

where $\Gamma(u, v)=\int_{0}^{v} x^{u-1} \exp (-x) \mathrm{d} x$ is the lower incomplete Gamma function. The expected durations $\mathbb{E}\left[T_{i j l}^{p}\right]$ and $\mathbb{E}\left[T_{i j l}^{c}\right]$ are evaluated to $\mathbb{E}\left[T_{i j l}^{p}\right]=\alpha_{i j l}^{p} \zeta_{i j l}^{p}$ and $\mathbb{E}\left[T_{i j l}^{c}\right]=\alpha_{i j l}^{c} \zeta_{i j l}^{c}$. In what follows, for $i \in\{1, \ldots, n\} ; j \in\left\{1, \ldots, N_{i}\right\}$ and $l \in\left\{1, \ldots, L_{i j}\right\}$, we assume that $\zeta_{i j l}=1$ and therefore combining Equations (10) and (11) together with the fact that $T_{i j 1}^{c}=T_{i j}^{m r}$, we have that:

$$
\begin{aligned}
\alpha_{i j 1}^{c} & =\mathbb{E}\left[T_{i j}^{m r}\right], \text { and } \\
\alpha_{i j l}^{p} & =\mathbb{E}\left[T_{i j}^{p}\right]\left(1-\theta_{i j l}\right)^{\delta_{i j}\left(A_{i j}\right)} \\
\alpha_{i j l}^{c} & =\mathbb{E}\left[T_{i j}^{c}\right]\left(1-\theta_{i j l}\right)^{\delta_{i j}\left(A_{i j}\right)} .
\end{aligned}
$$

Accordingly, we would like to note that for two random variables $W_{1}$ and $W_{2}$ each governed by a Gamma distribution $\operatorname{Gam}\left(\alpha_{1}, \zeta\right)$ and $\operatorname{Gam}\left(\alpha_{1}, \zeta\right)$, the sum $W_{1}+W_{2}$ is also governed by a Gamma distribution $\operatorname{Gam}\left(\alpha_{1}+\alpha_{2}, \zeta\right)$.

Using the above assumptions and observations, the chance constraint (Equation (24) in the SSMOP of Section 5) can be evaluated by determining the cdf of the total maintenance time $T M T=P M T+C M T$ incurred during the break by both PM and CM. To do so, let us first observe that from Equations (20) and (21), it follows that PMT and CMT both follow, respectively, the Gamma distributions $\operatorname{Gam}\left(\alpha_{p}, 1\right)$ and $\operatorname{Gam}\left(\alpha_{c}, 1\right)$ such that:

$$
\begin{aligned}
\alpha_{p} & =\sum_{i=1}^{n} \sum_{j=1}^{N_{i}} \sum_{l=2}^{L_{i j}} \alpha_{i j l}^{p} \cdot X_{i j} \cdot z_{i j l} \\
\alpha_{c} & =\sum_{i=1}^{n} \sum_{j=1}^{N_{i}} \sum_{l=1}^{L_{i j}} \alpha_{i j l}^{c} \cdot\left(1-X_{i j}\right) \cdot z_{i j l} .
\end{aligned}
$$


Table 1. Maintenance levels and their respective age reduction and adjustment coefficients values: case of Experiment \#1.

\begin{tabular}{lcccccccc}
\hline$l$ & 1 & 2 & 3 & 4 & 5 & 6 & 7 & 8 \\
\hline$\theta_{i j l}$ & 1 & 0.35 & 0.3 & 0.25 & 0.2 & 0.15 & 0.1 & 0 \\
$\xi_{i j l}$ & 1 & 1.35 & 1.3 & 1.25 & 1.2 & 1.15 & 1.1 & 1 \\
\hline
\end{tabular}

From the result of Equations (33)-(35), the above equations become:

$$
\begin{aligned}
\alpha_{p}= & \sum_{i=1}^{n} \sum_{j=1}^{N_{i}} \sum_{l=2}^{L_{i j}} \mathbb{E}\left[T_{i j}^{p}\right]\left(1-\theta_{i j l}\right)^{\delta_{i j}\left(A_{i j}\right)} \cdot X_{i j} \cdot z_{i j l}, \text { and } \\
\alpha_{c}= & \sum_{i=1}^{n} \sum_{j=1}^{N_{i}} \mathbb{E}\left[T_{i j}^{m r}\right] \cdot\left(1-X_{i j}\right) \cdot z_{i j 1}, \\
& +\sum_{i=1}^{n} \sum_{j=1}^{N_{i}} \sum_{l=2}^{L_{i j}} \mathbb{E}\left[T_{i j}^{c}\right]\left(1-\theta_{i j l}\right)^{\delta_{i j}\left(A_{i j}\right)} \cdot\left(1-X_{i j}\right) \cdot z_{i j l} .
\end{aligned}
$$

Finally, since $T M T=P M T+C M T$, it follows that $T M T$ is also governed by a Gamma distribution $\operatorname{Gam}(\alpha, 1)$ where $\alpha=\alpha_{p}+\alpha_{c}$ and the corresponding pdf and cdf are denoted, respectively, by $g(t)$ and $G(t)$ :

$$
\begin{aligned}
g(t) & =\frac{t^{\alpha_{p}+\alpha_{c}-1}}{\Gamma\left(\alpha_{p}+\alpha_{c}\right)} \exp (-t), \\
G(t) & =\frac{\Gamma\left(\alpha_{p}+\alpha_{c}, t\right)}{\Gamma\left(\alpha_{p}+\alpha_{c}\right)} .
\end{aligned}
$$

\subsection{Experiment \# 1: Analysis of a single component system}

This experiment shows how the stochasticity of the break, mission and maintenance durations impact the selective maintenance decisions. First, the case of a single component $P_{i j}$ is considered. The lifetime of this component is assumed to be Weibull distributed with shape and scale parameters set, respectively, to $\beta_{i j}=1.5$ and $\eta_{i j}=25$. For this component, a list of 9 maintenance levels $l \in\{0,1, \ldots, 8\}$ are available. Let us recall that the first maintenance level corresponds to the" $D o$ nothing" case, that the seconds maintenance level $l=1$ corresponds to a minimal repair action whose age reduction and adjustment coefficients are $\theta_{i j 1}=1$ and $\xi_{i j 1}=1$, that the highest level $l=L_{i j}=8$ is the replacement action whose age reduction and adjustment coefficients are $\theta_{i j 8}=0$ and $\xi_{i j 8}=1$. The value corresponding to the age reduction and adjustment coefficients $\theta_{i j l}$ and $\xi_{i j l}$ assigned to each intermediate maintenance level $l$ are given in Table 1.

The single component $P_{i j}$ is considered to have survived the mission that just ended (i.e. $X_{i j}=1$ ) with an age $A_{i j}=10$. The duration $U$ of the next mission is a truncated Normal distribution $\mathcal{N}(8,1.5)$ defined on the support [7, 12] with its corresponding pdf drawn in Figure 3. The required minimum reliability level $\mathcal{R}_{0}$ for the next mission is set to $\mathcal{R}_{0}=70 \%$.

Since the component is still functioning at the end of the current mission, only preventive replacement $\operatorname{cost} C_{i j}^{p}$ and corresponding preventive replacement duration $T_{i j}^{p}$ are computed. The preventive replacement cost is set to $C_{i j}^{p}=10$. Hence, the $\operatorname{cost} C_{i j l}^{p}$ for each intermediate maintenance level $l=2, \ldots, 8$ are obtained according to Equation (4). The preventive replacement duration $T_{i j}^{p}$ is a random variable governed by a Gamma distribution $\operatorname{Gam}\left(\alpha_{i j l}^{p}, 1\right)$ and its corresponding expected value $\mathbb{E}\left[T_{i j}^{p}\right]=\alpha_{i j l}^{p}$ is set to $\mathbb{E}\left[T_{i j}^{p}\right]=2.5$. The expected duration values $\mathbb{E}\left[T_{i j l}^{p}\right]$ for intermediate maintenance levels can be derived from Equation (10). The maintenance level $l=1$ corresponding to a minimal repair action is not eligible since the component under consideration is still functioning at the end of the current mission.

In what follows, we compare and analyse the results of the optimisation problem obtained in the deterministic case (average durations) and stochastic case (full distribution of the durations).

Let us first evaluate the reliability of the component to complete the next mission in terms of each maintenance level. The results obtained are given in Table 2 for both the deterministic and random (stochastic) mission duration. In the stochastic case, the reliability values are computed according to Equation (13). In this table, maintenance level $l=0$ corresponds to the 'Do nothing' case. This table also displays the difference in reliability between the deterministic and stochastic approaches. One may indeed observe that the reliability error rises beyond $2 \%$ for the instance considered. 
Table 2. Component reliability results obtained from deterministic and stochastic cases of mission duration $U$ : case of Experiment \#1.

\begin{tabular}{lccc}
\hline $\begin{array}{l}\text { Maintenance } \\
\text { level } l\end{array}$ & $\begin{array}{c}\text { Reliability in } \% \\
\text { (deterministic) }\end{array}$ & $\begin{array}{c}\text { Reliability in }(\% \\
\text { (stochastic) }\end{array}$ & $\begin{array}{c}\text { Error in } \\
\text { Reliability\% }\end{array}$ \\
\hline 0 & 69.91 & 67.77 & 2.14 \\
1 & - & - & - \\
2 & 70.44 & 68.08 & 2.36 \\
3 & 72.23 & 71.81 & 2.30 \\
4 & 74.03 & 73.70 & 2.22 \\
5 & 75.85 & 75.62 & 2.15 \\
6 & 77.69 & 77.57 & 1.97 \\
8 & 79.55 & 81.63 & 1.81 \\
\hline
\end{tabular}

Given the required minimum reliability level $\mathcal{R}_{0}=70 \%$, the no maintenance level $(l=0)$ results in a reliability which is not feasible in both stochastic and deterministic cases. This leads to the absolute need to improve the component reliability. In the deterministic case, selecting maintenance level $l=2$ is sufficient to meet the required reliability for the next mission. Indeed, performing a maintenance of level 2 results in a reliability value of $70.44 \%$ which is greater than the minimum required reliability of $70 \%$. However, with a maintenance level 2 , the resulting reliability value in the stochastic case is only $68.08 \%$, which is rather insufficient to meet the required minimum reliability level. The stochastic case suggests to perform a PM action with level at least equal to $l=4$. This minimum maintenance level results in a component reliability improvement of $4.04 \%$ when compared to the level 0 option. Once again, this small case demonstrates the fact that it is more effective to consider the more realistic stochastic aspect of mission durations when dealing with maintenance optimisation problem in general, and in selective maintenance optimisation problem in particular.

Let us now consider the additional chance constraint represented by Equation (24) in the SSMOP. Let us assume that the pdf $f_{D}(t)$ and cdf $F_{D}(t)$ of the stochastic break duration $D$ are defined on a support $\left[D_{\min }, D_{\max }\right]=[1,4]$ meaning that the break duration lies between $D_{\min }=1$ and $D_{\max }=4$. In the present example, we also assume that $D$ is governed by a truncated Normal distribution $\mathcal{N}(1.4,0.5)$, its corresponding pdf is drawn in Figure 4 . The average value of the break duration is $\mathbb{E}[D]=1.58$ which is greater than the average maintenance duration $\mathbb{E}\left[T_{i j l}^{p}\right]=1.53$ required to carry out the PM action of level $l=4$. On average, the PM action of level 4 can be executed during the break. However, if the stochastic approach is considered, the chance to carry out this PM action is computed as the probability $\operatorname{Pr}\left(D \geq T_{i j l}^{p}\right)$ where $l=4$ such that:

$$
\operatorname{Pr}\left(T_{i j l}^{p} \leq D\right)=\int_{D_{\min }}^{D_{\max }} G_{i j l}^{p}(t) f_{D}(t) \mathrm{d} t
$$

Using Equation (31), we then have:

$$
\operatorname{Pr}\left(T_{i j l}^{p} \leq D\right)=\frac{1}{\Gamma\left(\alpha_{i j l}^{p}\right)} \int_{D_{\min }}^{D_{\max }} \Gamma\left(\alpha_{i j l}^{p}, t\right) f_{D}(t) \mathrm{d} t .
$$

The above integral is evaluated to $61.17 \%$, meaning that the maintenance action of level $l=4$ is a feasible solution of the stochastic selective optimisation problem if and only if the service ratio level $\tau_{s}$ is set to a value less than or equal to $61.17 \%$ $\left(\tau_{s} \leq 61.17 \%\right)$. There still exists a $38.83 \%$ risk that the maintenance action will not be performed. Once again, this simple example clearly demonstrates how the stochasticity of the break duration may also impact the maintenance decisions.

\subsection{Experiment \# 2: Analysis of a multi-component system}

In this second experiment, the impact of stochastic mission and break durations as well as that of the maintenance times on selective maintenance decisions is studied for a series-parallel system $S$. This system is composed of two series subsystems $S_{i}$, where the first is composed of $N_{1}=3$ parallel components $P_{1 j}(j=1,2,3)$, while the second contains $N_{2}=2$ components $P_{2 j}(j=1,2)$ in parallel. The failure times of the system component $P_{i j}$ follow a Weibull distribution whose shape and scale parameters are, respectively, given by $\beta_{i j}$ and $\eta_{i j}\left(i=1,2\right.$ and $\left.j=1, \ldots, N_{i}\right)$. Values of these parameters are shown in Table 3. This table shows also the value of $A_{i j}$ corresponding to the age of components $P_{i j}$ at the end of the current mission, in addition to the value of the state variables $X_{i j}$ corresponding to the operating state (functioning or failed) of 


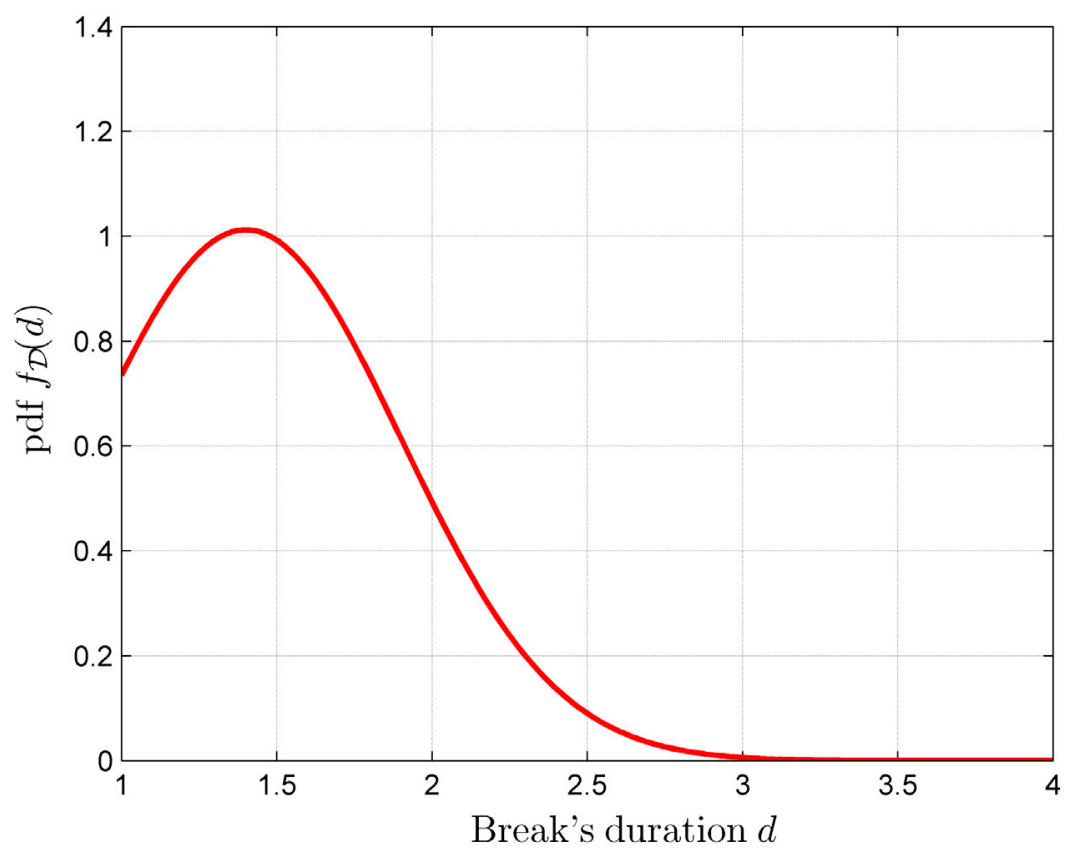

Figure 4. Plot of the pdf of the break duration: case of Experiment \#1.

Table 3. Components' lifetimes parameters and their corresponding current operating states and age: case of Experiment \#2.

\begin{tabular}{lcccc}
\hline$P_{i j}$ & $\beta_{i j}$ & $\eta_{i j}$ & $X_{i j}$ & $A_{i j}$ \\
\hline$P_{11}$ & 3 & 22.5 & 1 & 5 \\
$P_{12}$ & 2.8 & 30 & 0 & 7.5 \\
$P_{13}$ & 2.5 & 30 & 0 & 5 \\
$P_{21}$ & 1.75 & 22.5 & 1 & 5 \\
$P_{22}$ & 1.8 & 30 & 0 & 7.5 \\
\hline
\end{tabular}

Table 4. Maintenance levels and their respective age reduction and adjustment coefficients values: case of Experiment \#2.

\begin{tabular}{lllllllll}
\hline$l$ & 1 & 2 & 3 & 4 & 5 & 6 & 7 \\
\hline$\theta_{i j l}$ & 1 & 0.35 & 0.3 & 0.25 & 0.2 & 0.15 & 0.1 \\
$\xi_{i j l}$ & 1 & 1.25 & 1.2 & 1.15 & 1.1 & 1.05 & 1.02 & 1 \\
\hline
\end{tabular}

components. According to this table, only components $P_{11}$ and $P_{21}$ survived the current mission $\left(X_{11}=X_{21}=1\right)$ while the other components are in the failed state.

In this experiment, all components are assigned in the same list of nine potential maintenance levels $\{0,1, \ldots, 8\}$. The values corresponding to the age reduction and adjustment hazard rate coefficients $\theta_{i j l}$ and $\xi_{i j l}$ corresponding maintenance level $l \geq 1$ are given in Table 4. Table 5 gives the time $T_{i j}^{m r}$ and the cost $C_{i j}^{m r}$ of a minimal repair as well as the durations $T_{i j}^{p}$, $T_{i j}^{c}$, and the costs $C_{i j}^{p}$ and $C_{i j}^{c}$ of preventive and corrective replacements, respectively. Costs corresponding to the intermediate maintenance levels are obtained via Equations (3)-(5), while their durations are derived from Equations (9)-(11).

For the analysis below, we assume that the duration $U$ of the next mission is stochastic and governed by a truncated Normal distribution $\mathcal{N}(16,2.5)$ on the support [14,24] (its pdf is drawn in Figure 5). The duration $D$ of the break is assumed to follow a truncated Normal distribution $\mathcal{N}(8,0.5)$ on the support $[4,10]$ (its pdf is drawn in Figure 6). We also set the required minimum level of the system reliability $\mathcal{R}_{0}$ for the next mission to $\mathcal{R}_{0}=82 \%$. We also require that the optimal maintenance plan should be completed with a minimum required probability of $\tau_{s}=75 \%$. 
Table 5. Values of times and costs of minimal repair and that of preventive and corrective replacements: case of Experiment \#2.

\begin{tabular}{ccccccc}
\hline$P_{i j}$ & $\mathbb{E}\left[M R T_{i j}\right]$ & $M R C_{i j}$ & $\mathbb{E}\left[R T_{i j}^{p}\right]$ & $R C_{i j}^{p}$ & $\mathbb{E}\left[R T_{i j}^{c}\right]$ & $R C_{i j}^{c}$ \\
\hline$P_{11}$ & 0.8 & 5 & 0.8 & 70 & 2.4 & 80 \\
$P_{12}$ & 0.8 & 6 & 0.8 & 75 & 2.4 & 85 \\
$P_{13}$ & 1.2 & 6 & 0.4 & 50 & 3.2 & 70 \\
$P_{21}$ & 1.2 & 6 & 0.4 & 60 & 3.2 & 70 \\
$P_{22}$ & 1.2 & 5 & 0.41 & 60 & 3.2 & 70 \\
\hline
\end{tabular}

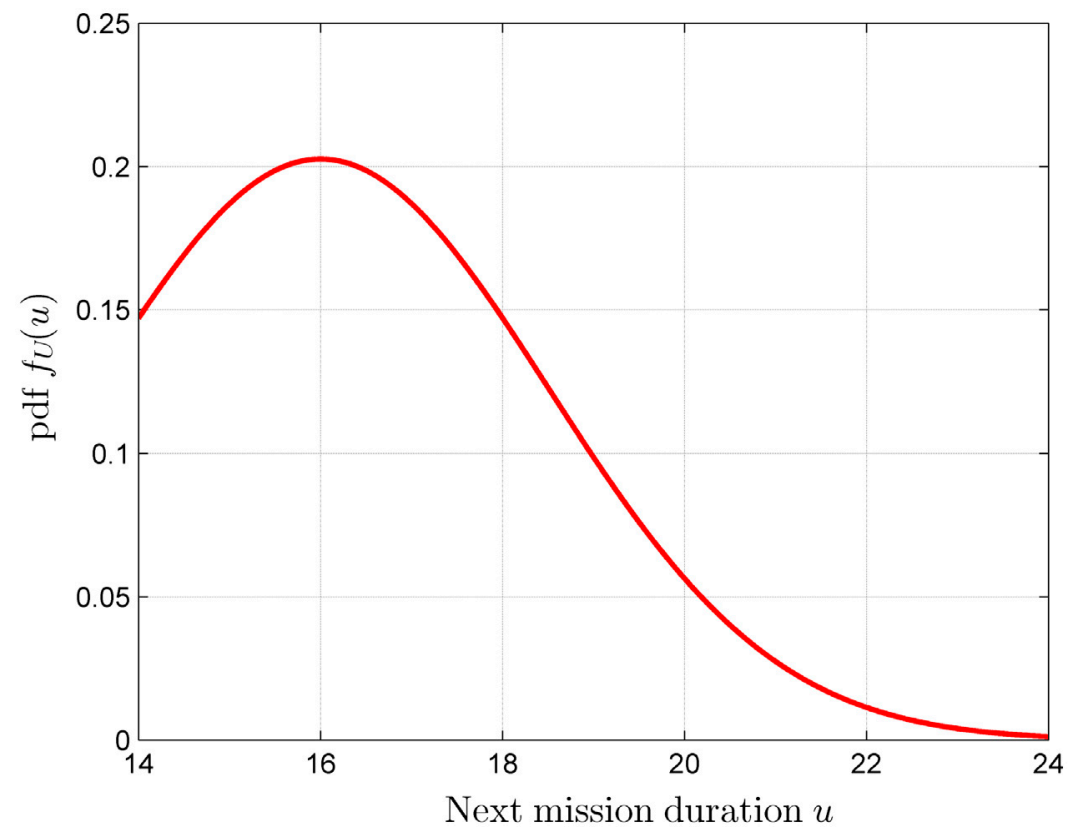

Figure 5. The pdf corresponding to the duration of the next mission: case of Experiment \#2.

If no maintenance is performed on the system components, the probability of the system successfully completing the next mission is evaluated to $\mathcal{R}^{c}=16.85 \%$ in the stochastic case and equal to $\mathcal{R}^{c}=19.86 \%$ in the deterministic case. In both cases, reliability values are indeed lower than that required to execute the mission. Solving the selective maintenance problem is therefore needed to improve the system reliability.

Given the required minimum reliability level $\mathcal{R}_{0}$ together with the limited stochastic time $D$ of the break, if the selective maintenance problem is solved by assuming the deterministic duration of the next mission (i.e. the next mission duration is set to 16 which is the deterministic value of $U$ ), in this case the optimal solution suggests the following selective maintenance plan. All system components, except component $P_{11}$, are selected to receive a maintenance action. A minimal repair is carried out on component $P_{12}$. Components $P_{13}$ and $P_{22}$ are selected to undergo CM actions of levels 4 and 7, respectively, while component $P_{21}$ receives a PM of level 7. This maintenance plan is likely to be completely performed during the break with probability $85.11 \%$. The resulting system reliability and the expected total cost induced by maintenance actions are, respectively, $\mathcal{R}^{c}=82.04 \%$ and $T M C=120.51$.

Applying the above maintenance plan in the case where the duration of the next mission is stochastic leads to a system reliability equal to $78.63 \%$ which is indeed less than the required minimum reliability level $\mathcal{R}_{0}=82 \%$. In the stochastic case, this selective maintenance plan is unfortunately unable to allow the system reliability to reach the required minimum level for the next mission. Thus, if for some reason, the mission duration is extended, there will be then a high risk to operate the mission with the required reliability level.

If this same selective maintenance problem is now solved while considering the duration of the next mission to be stochastic, the following selective maintenance plan is obtained according to which components $P_{11}$ is selected to receive a PM of levels 5. Furthermore, a preventive replacement is performed on component $P_{21}$, a minimal repair is executed on 


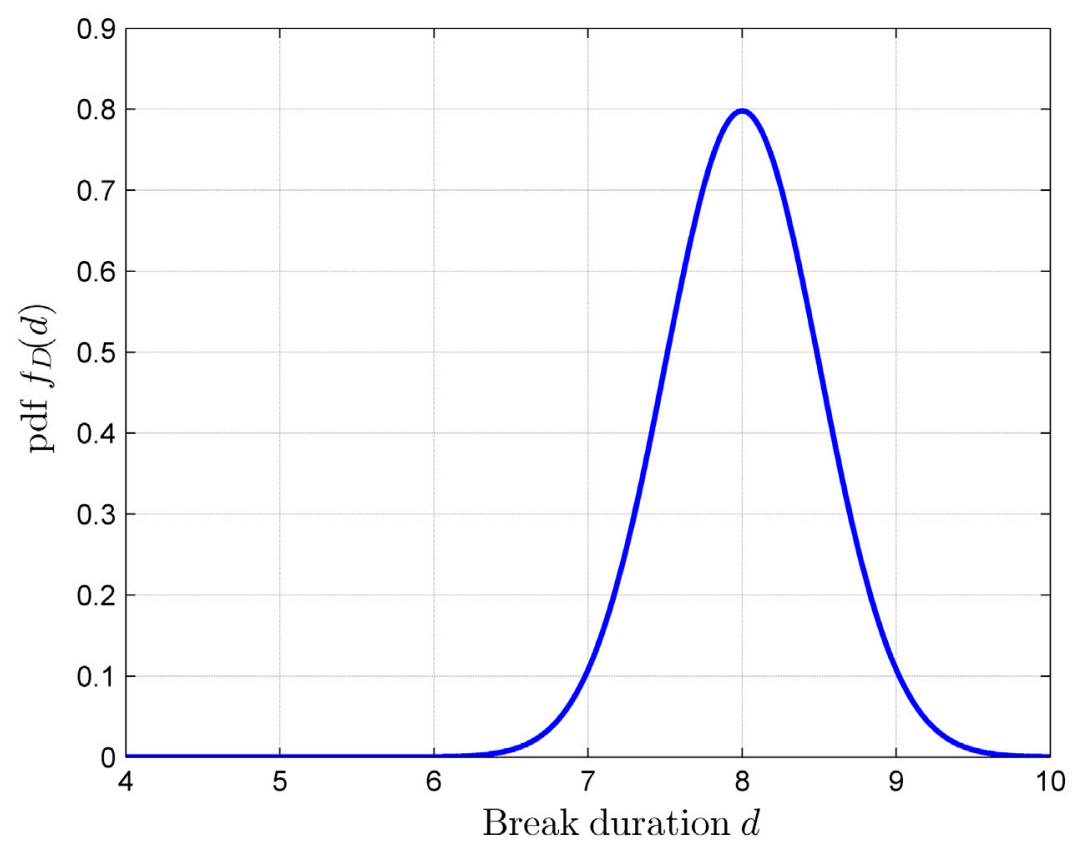

Figure 6. The pdf corresponding to the break duration: case of Experiment \#2.

component $P_{13}$, while CM of levels 5 and 7 are, respectively, carried out on components $P_{12}$ and $P_{22}$. This maintenance plan could be achieved with probability $\tau_{s}=76.07 \%$. The resulting system reliability is $82.05 \%$. The total cost induced by this selective maintenance plan is $T M C=200.84$.

\section{Conclusion}

This paper addressed the selective maintenance optimisation problem for a multi-component system. For each component of the system, a list of maintenance actions is available from minimal repair to replacement through imperfect maintenance actions. The more general imperfect maintenance model, namely the hybrid hazard rate model is used to characterise the reliability improvement for the components in the system. The system performs several missions separated by scheduled breaks during which maintenance activities can take place. Durations of both breaks and missions are considered as random variables governed by appropriate probability distributions. Furthermore, maintenance durations are also considered stochastic and follow given probability distributions. All these distributions are fully accounted for and integrated in the selective maintenance formulation resulting in a non-linear stochastic optimisation programme.

We demonstrated the importance of solving the selective maintenance problem as a stochastic optimisation programme and illustrated this approach on a series-parallel system. Experiments are conducted and their results are discussed. Comparisons are provided between the proposed approach and earlier approaches where both break and mission durations are assumed deterministic. The proposed stochastic selective maintenance problem provides more effective solutions in terms of risks related to the execution of the selected maintenance plan. Furthermore, considering mission durations as stochastic rather than deterministic allows efficient and accurate evaluation of the system probability to successfully complete the next mission.

Future extensions include the investigation of new exact and heuristics approaches to solve the large-scale version of the problem investigated in this paper. Additional extensions should target more complex multi-component configurations such as the $k$-out-of- $n$ structures. Studying trade-off between several objectives while considering multiple repair resources is also a major importance issue to deal with for selective maintenance.

\section{Disclosure statement}

No potential conflict of interest was reported by the authors.

\section{ORCID}

Claver Diallo (D) http://orcid.org/0000-0002-7381-2187 


\section{References}

Aggrawal, K. K. 1976. "Redundancy Optimization in General Systems." IEEE Transactions on Reliability R-25 (25): $330-332$.

Birge, J. R., and F. Louveaux. 1997. Introduction to Stochastic Programming. New York, NY: Springer Series in Operations Research and Financial Engineering, Springer.

Canfield, R. V. 1986. “Cost Optimization of Periodic Preventive Maintenance.” IEEE Transactions on Reliability 35 (1): $78-81$.

Cassady, C. R., W. P. Murdock, and E. A. Pohl. 2001. "Selective Maintenance for Support Equipement Involving Multiple Maintenance Actions." European Journal of Operational Research 129: 252-258.

Cassady, C. R., E. A. Pohl, and W. P. Murdock. 2001. "Selective Maintenance Modeling for Industrial Systems.” Journal of Quality in Maintenance Engineering 7 (2): 104-117.

Chen, C, M. Q.-H. Mend, and M. J. Zuo. 1999. "Selective Maintenance Optimization for Multi-state Systems.” Proceedings of the IEEE Canadian Conference on Electrical and Computer Engineering, Edmonton, Canada.

Dao, C. D., and M. J. Zuo. 2016. "Selective Maintenance for Multi-state Series Systems with s-dependent Components." IEEE Transactions on Reliability 65 (2): 525-539.

Dao, C. D., and M. J. Zuo. 2017. "Selective Maintenance of Multi-state Systems with Structural Dependence." Reliability Engineering and System Safety 159: 184-195.

Dao, C. D., M. J. Zuo, and M. Pandey. 2014. "Selective Maintenance for Multi-state series-parallel Systems under Economic Dependence." Reliability Engineering and System Safety 121: 240-249.

Djelloul, I, A. Khatab, E.-H. Aghezzaf, and Z. Sari. 2015. “Optimal Selective Maintenance Policy for Series-parallel Systems Operating Missions of Random Durations." International conference on Computers \& Industrial Engineering (CIE 45), Metz, France.

El-Ferik, S., and M. Ben-Daya. 2006. "Age-based Hybrid Model for Imperfect Preventive Maintenance.” IIE Transactions 38: $365-375$.

Gopal, K., K. K. Aggrawal, and J. S. Gupta. 1978. “An Improved Algorithm for Reliability Optimization.” IEEE Transactions on Reliability R-27 (27):325-328.

Khatab, A. 2015. "Hybrid Hazard Rate Model for Imperfect Preventive Maintenance of Systems Subject to Random Deterioration.” Journal of Intelligent Manufacturing 26 (3): 601-608.

Khatab, A., and E.-H. Aghezzaf. 2016. "Selective Maintenance Optimization When Quality of Imperfect Maintenance Actions are Stochastic.” Reliability Engineering \& System Safety 150: 182-189.

Khatab, A., E.-H. Aghezzaf, I. Djelloul, and Z. Sari. 2016. "Selective Maintenance for Series-parallel Systems When Durations of Missions and Planned Breaks are Stochastic." 8th IFAC Conference on Manufacturing Modelling, Management, and Control (MIM 2016), Troyes, France.

Khatab, A., D. Ait-Kadi, and M. Nourelfath. 2007. "Heuristic-based Methods for Solving the Selective Maintenance Problem for Seriesprallel Systems.” International Conference on Industrial Engineering and Systems Management, Beijing, China.

Khatab, A., N. Rezg, and D. Ait-Kadi. 2011a. "Optimal Replacement with Minimal Repair Policy for a System Operating over a Random Time Horizon." Journal of Quality in Maintenance Engineering 17 (4): 415-423.

Khatab, A., N. Rezg, and D. Ait-Kadi. 2011b. “Optimum Block Replacement Policy over a Random Time Horizon." Journal of Intelligent Manufacturing 22 (6): 885-889.

Kijima, M. 1989. “Some Results for Repairable Systems with General Repair.” Journal of Applied probability 26 (1): 89-102.

Kijima, M., H. Murimura, and Y. Suzuki. 1988. "Periodical Replacement Problem without Assuming Minimal Repair.” European Journal of Operational Research 37 (2): 194-203.

Lam, Y. 1988. “Geometric Processes and Replacement Problem.” Acta Mathematicae Applictae Sinica 4 (4): $366-377$.

Lin, D., M. J. Zuo, and R. C. M. Yam. 2000. "General Sequential Imperfect Preventive Maintenance Models.” International Journal of Reliability, Quality and Safety Engineering 7 (3): 253-266.

Lin, D., M. J. Zuo, and R. C. M. Yam. 2001. "Sequential Imperfect Preventive Maintenance Models with Two Categories of Failure Modes." Naval Research Logistics 48: 172-183.

Liu, Y., and H.-Z. Huang. 2010. "Optimal Selective Maintenance strategy for Multi-state Systems under Imperfect Maintenance." IEEE Transactions on Reliability 59 (2): 356-367.

Lust, T., O. Roux, and F. Riane. 2009. "Exact and Heuristic Methods for the Selective Maintenance Problem." European Journal of Operational Research 197: 1166-1177.

Maaroufi, G., A. Chelbi, and N. Rezg. 2013. "Optimal Selective Renewal for Systems Subject to Propoagated Failures with global effect and Failure Isolation Phenomena." Reliability Engineering and System Safety 114: 61-70.

Malik, M. A. K. 1979. "Reliable Preventive Maintenance Scheduling. AIIE.” Transactions 11 (3): 221-228.

Nakagawa, T. 1988. "Sequential Imperfect Preventive Maintenance Policies.” IEEE Transactions on Reliability 37 (3): $295-308$.

Nakagawa, T. 2008. Advanced Reliability Models and Maintenance Policies, London: Springer.

Nakagawa, T., and S. Mizutani. 2009. “A Summary of Maintenance Policies for a Finite Interval.” Reliability Engineering and System Safety 94 (1): 89-96.

Panday, M., M. J. Zuo, R. Moghaddass, and M. K. Tiwari. 2013. "Selective Maintenance for Binary Systems under Imperfect Repair." Reliability Engineering and System Safety 113: 42-51.

Pandey, M., M. J. Zuo, and R. Moghaddass. 2013. "Selective Maintenance Modeling for Multistate System with Multistate Components under Imperfect Maintenance.” IIE Transcations 45: 1221-1234. 
Park, M., and H. Pham. 2010. "Altered Quasi-renewal concepts for Modeling Renewable Warranty Costs with Imperfect Repairs." Mathematical and Computer Modelling 52: 1435-1450.

Pham, H., and W. Wang. 1996. "Imperfect Maintenance." European Journal of Operational Research 94: 425-438.

Rajagopalan, R., and C. R. Cassady. 2006. “An Improved Selective Maintenance Solution Approach.” Journal of Quality in Maintenance Engineering 12 (2): 172-185.

Rice, W. F., C. R. Cassady, and J. A. Nachlas. 1998. “Optimal Maintenance Plans under Limited Maintenance Time.” Proceedings of Industrial Engineering Conference, Banff, BC, Canada.

Sharma, J., and K. V. Venkateswaran. 1971. "Redundancy Optimization in General Systems." IEEE Transactions on Reliability R-20 (20): 256-259.

Sheu, S.-H., and C.-C. Chang. 2009. “An Extended Periodic Imperfect Preventive Maintenance Model with Age-Dependent Failure Type.” IEEE Transactions on Reliability 58 (2): 397-405.

Spall, J. C. 2005. Introduction to Stochastic Search and Optimization: Estimation, Simulation, and Control. Wiley Series in Discrete Mathematics and Optimization, Hoboken, NJ: Wiley.

Tanwar, M., R. N. Rai, and N. Bolia. 2014. "Imperfect Repair Modeling Using Kijima Type Generalized Renewal Process.” Reliability Engineering \& System Safety 124: 24-31.

Wang, H. 2002. “A Survey of Maintenance Policies of Deteriorating Systems.” European Journal of Operational Research 139: 469-489.

Wang, H., and H. Pham. 1999. "Some Maintenance Models and Availability with Imperfect Maintenance in Production Systems.” Annals of Operations Research 91: 305-318.

Wang, H. Z., and H. Pham. 2006. Reliability and Optimal Maintenance. London: Springer.

Won, Y. Y., and H. C. Chung. 2000. "Optimum Replacement Intervals with Random Time Horizon.” Journal of Quality in Maintenance Engineering 6 (4): 269-274.

Wu, S., and D. Clements-Croome. 2005. "Preventive Maintenance Models with Random Maintenance Quality." Reliability Engineering and System Safety 90: 99-105.

Zhou, Y., T. R. Lin, Y. Sun, Y. Bian, and L. Ma. 2015. “An Effective Approach to Reducing Strategy Space for Maintenance Optimisation of Multistate Series-parallel systems.” Reliability Engineering and System Safety 138: 40-53.

Zhu, H., F. Liu, X. Shao, Q. Liu, and Y. Deng. 2011. "A Cost-based Selective Maintenance Decision-making Method for Machining Line.” Quality and Reliability Engineering International 27: 191-201. 